\title{
Microbiology of Urinary Tract Infection and the Status of Urinary Isolates in Pregnant Women
}

\author{
Sharmila Parajuli ${ }^{1}$, Thapa $\mathrm{B}^{2}$ \\ ${ }^{1}$ Department of Pathology, Kathmandu Model Hospital, Nepal \\ ${ }^{2}$ Department of Pathology, Valley Maternity Hospital, Putalisadak, Kathmandu.
}

\begin{abstract}
Introduction: Urinary tract infection (UTI) is one of the most frequently encountered problems owing to significant number of patients needing hospitalization during pregnancy. The incidence of UTI in pregnant women is reported to be high up to $7-8 \%$.

Methods: This is a prospective study conducted in Valley Maternity Hospital during a period of 6 months (Jan 2011 to June 2011). 520 MSU (Mid stream urine samples) from pregnant women clinically suspected of urine infection were evaluated by urine dipstick analysis, microscopic and culture method. The isolates were identified and antibiotic sensitivity pattern was determined by standard protocol.
\end{abstract}

Results: The majority of the patients were in-between the age group of $20-30$ years- 338cases (65\%) and these patients usually presented in the first trimester of pregnancy- 317cases $(60.96 \%)$. Out of the 520 clinically suspected UTI cases, 232 (44.61\%) was culture positive. Out of the culture positive cases; Escherichia coli (E.coli) was the most common accounting for a total of 144cases $(80 \%)$. Nitrofurantoin was found to be the most effective drug against the gram negative (Gm-ve) bacteria. Similarly, Ampicillin, Amoxycillin and Cloxacillin were found to be effective agent against gram positive $(\mathrm{Gm}+\mathrm{ve})$ bacteria.

Conclusions: Screening for bacteriuria is recommended among all pregnant women at the first prenatal visit and in the subsequent trimesters of pregnancy. Prompt treatment of symptomatic UTI and asymptomatic bacteriuria is required in pregnant women to avoid complications like preterm birth, low birth weight and increased perinatal mortality.

Keywords: UTI; pregnant women; antibiotics; culture method.

\section{INTRODUCTION}

Urinary tract infection (UTI) is one of the most frequently encountered problems owing to significant number of patients needing hospitalization during pregnancy. The incidence of UTI in pregnant women is reported to be high upto $7-8 \%{ }^{1}$.

The frequency of asymptomatic bacteriuria occurs in $2-7 \%$ of pregnancies, similar to the non-pregnant population. However, upto $40 \%$ of these may progress to symptomatic upper tract UTI or pyelonephritis, significantly more in pregnant women ${ }^{1,2}$.
$2 \%$ of 24000 patients acquire acute pyelonephritis during pregnancy. UTI is associated with risks to both the fetus and mother including pyelonephritis, preterm birth, low birth weight and increased perinatal mortality ${ }^{3}$.

Several physiologic changes during pregnancy cause otherwise healthy women to be more susceptible to serious sequelae from urinary tract infections. The infections can be symptomatic or asymptomatic. Treatment of asymptomatic bacteriuria reduces the risk of a symptomatic infection ${ }^{4,5}$.

Correspondence:

Dr. Sharmila Parajuli

Department of Pathology, Kathmandu Model Hospital, Nepal.

Email:drsharmi@hotmail.com 
Presentation varies depending on whether the patient has asymptomatic bacteriuria, a lower tract UTI (cystitis) and an upper tract UTI (pyelonephritis). Burning with urination (dysuria) is the most significant symptom in pregnant women with symptomatic cystitis. Symptoms of pyelonephritis include: fever associated with chills, nausea and vomiting, costovertebral angle (CVA) or flank pain ${ }^{6,7}$.

E. coli is the most common cause of UTI, accounting for $80-90 \%$ of cases. It originates from fecal flora that colonise the periurethral area (ascending infection) ${ }^{8}$, 9. Klebsiella, Enterobacter and Proteus species cause most of the remaining cases. Gram positive organisms, particularly Enterococcus fecalis and group B streptococcus are also clinically important pathogens ${ }^{10}$, 11 .

This article deals with the frequency of UTI in pregnant women, efficacy of detecting UTI by culture, significance of pus cells, etiological agents for UTI in pregnant women and their antibiotic sensitivity patterns.

\section{METHODS}

This is a prospective study conducted in Valley maternity hospital from Jan 2011- June 2011 (6 months). Verbal consent was taken to all the patients for fulfilling semi-structured interview questionnaire.

Purposive sample were selected on the basis of symptoms of increased frequency of micturition, dysuria, urgency and fever in clinically suspected UTI patients. All clinically suspected pregnant women of UTI, attending out patients department (OPD) and hospitalized were included whereas non-pregnant women were excluded.

520 MSU specimens were received in the lab. All specimens were investigated by conventional semiquantitative culture technique, pus cell count, RBC count, and epithelial cell count and albumin test. For culture, $0.001 \mathrm{ml}$ of uncentrifused mixed MSU specimens, were streaked onto Mac- Conkey agar and Blood agar plates with the help of standard flame sterilized loop. Bacterial growths were observed following overnight incubation and their sensitivity pattern were determined by Kirby-Bauer disc diffusion method.

\section{RESULTS}

Out of 520 clinically suspected pregnant women, the majority of the patients were in-between the age group of 20-30years, 338cases (65\%).

Out of the 520 clinically suspected UTI cases, 232 (44.61\%) was culture positive. The highest incidence of culture positivity was found to be $60.54 \%$ in the age group of 20-30 years.

Table 1: Age-wise distribution of cases along with culture positivity

\begin{tabular}{|l|l|l|l|}
\hline Age of patient & $<\mathbf{2 0}$ years & $\mathbf{2 0 - 3 0}$ years & $>\mathbf{3 0}$ years \\
\hline $\begin{array}{l}\text { Total suspected cases } \\
(\mathbf{N = 5 2 0})\end{array}$ & 150 & 338 & $32(5.67 \%)$ \\
\hline $\begin{array}{l}\text { Total culture positive } \\
\text { cases }\end{array}$ & 73 & 140 & 19 \\
$\mathbf{( 2 3 2 = 4 4 . 6 1 \% )}$ & $(31.29 \%)$ & $(60.54 \%)$ & $(8.16 \%)$ \\
\hline
\end{tabular}

Table 2. Cases of UTI according to the trimester of pregnancy

\begin{tabular}{|l|l|l|l|}
\hline Trimester & First & Second & Third \\
\hline Total cases & 317 & 37 & 166 \\
$(\mathbf{N}=\mathbf{5 2 0})$ & $(60.96 \%)$ & $(7.11 \%)$ & $(31.92 \%)$ \\
\hline
\end{tabular}

The majority of the patients presented in the first trimester of pregnancy- 317 cases

$(60.96 \%)$ followed by third trimester of pregnancy166 cases $(31.92 \%)$.

Table 3. Showing relationship of cases with pus cells and positive culture results

\begin{tabular}{|l|l|l|}
\hline $\begin{array}{l}\text { Pus cells/ } \\
\text { HPF }\end{array}$ & $\begin{array}{l}\text { Total suspected } \\
\text { cases (\%) }\end{array}$ & $\begin{array}{l}\text { Culture positive cases } \\
(\mathbf{\%})\end{array}$ \\
\hline $\mathbf{0}$ & $8(1.5 \%)$ & $0(0 \%)$ \\
\hline $\mathbf{0 - 1}$ & $197(37.88 \%)$ & $29(14.72 \%)$ \\
\hline $\mathbf{1 - 5}$ & $240(46.15 \%)$ & $128(53.33 \%)$ \\
\hline $\mathbf{5 - 1 0}$ & $75(14.42 \%)$ & $75(100 \%)$ \\
\hline
\end{tabular}

Out of the total suspected cases; $53.33 \%$ were urine culture positives and had pus cell counts (1-5/hpf)

However, cases which have more than 5 pus cells/ hpf were all culture positives. 
Figure 1. Gram stain frequency.

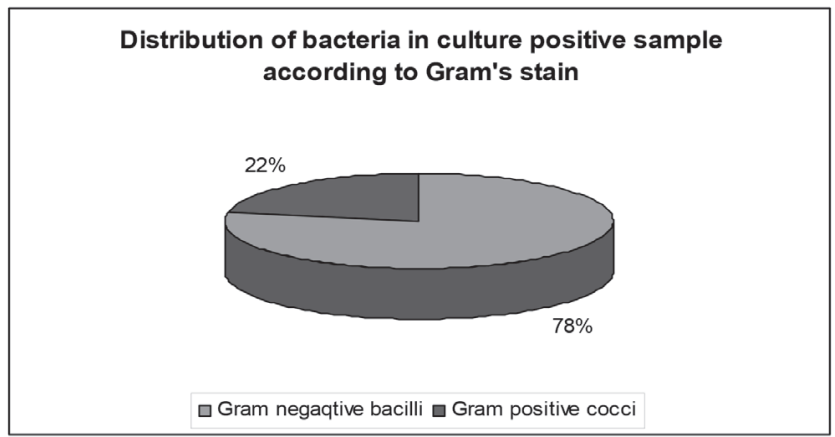

Out of the 132 culture positive cases, the most common organism isolated was Gram negative bacilli- 180 cases (78\%) followed by Gram positive cocci- 52 cases $(22 \%)$

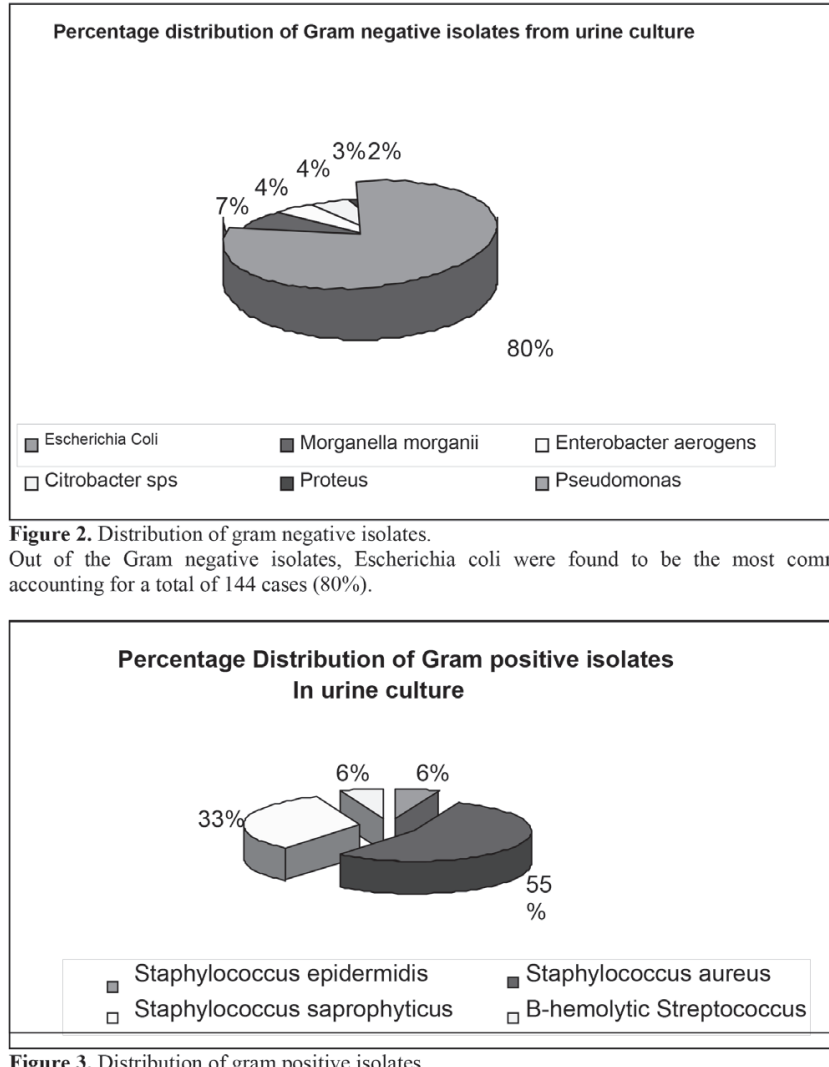

Amongst the Gm positive isolates, Staphylococcus aureus was the commonest accounting for a total of 29 cases $(55 \%)$.
Figure 4. Antibiotic sensitivity pattern of Gram negative bacteria from urine sample

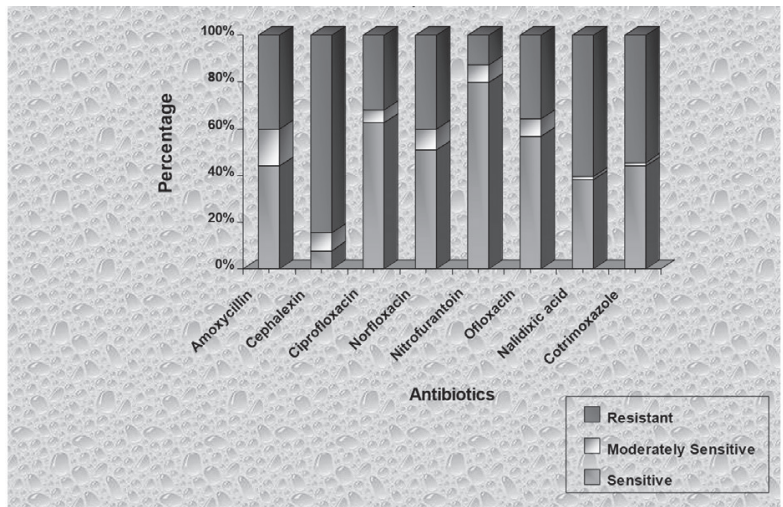

Nitrofurantoin was the most sensitive drug isolated with a sensitivity of $80 \%$. The least sensitive drug was cephalexin.

Figure 5. Antibiotic sensitivity pattern of Gram positive bacteria from urine sample

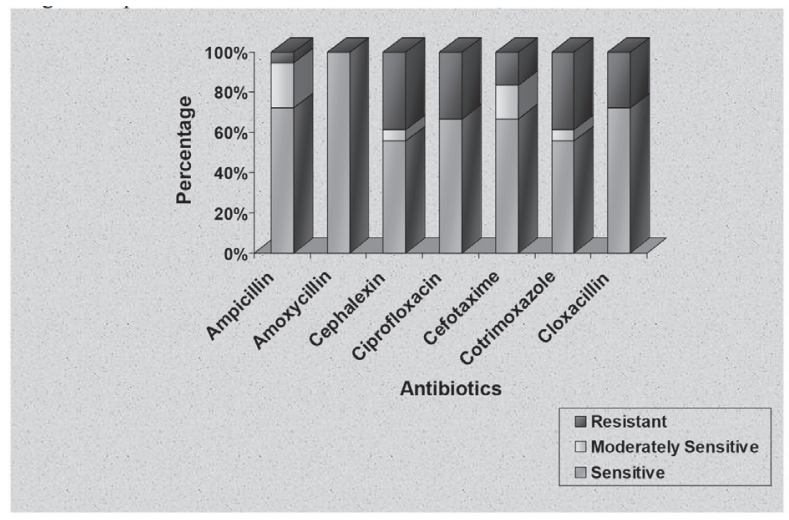

Amoxycillin, Ampicillin and cloxacillin were the sensitive drugs isolated against the $\mathrm{Gm}$ positive organisms.

\section{DISCUSSION}

UTI is very common during pregnancy. The majority of the patients in the present study were in-between the age group of $20-30$ years- 338 cases $(65 \%)$. This correlates well with a study conducted by Subedi M et al who showed the similar incidence as $61 \% .{ }^{12}$ This is explained by the fact that the highest incidence of pregnancy in Nepalese women is usually between 20-30years. 
Vaginal infections can cause or mimic UTI, which are common in women of reproductive age, affecting 25 $35 \%$ of women aged $20-40$ years. ${ }^{13}$

The majority of the patients in our study presented in the first trimester of pregnancy- 317cases $(60.96 \%)$ followed by third trimester of pregnancy- 166cases $(31.92 \%)$. This can be explained by the fact that all pregnant women in their first presentation are thoroughly screened with investigation measures including hematological profile, biochemical profile, viral markers and urine examination. This helps in detecting asymptomatic bacteriuria in the first trimester of pregnancy. ${ }^{14}$

Out of the total suspected cases; $53.33 \%$ were urine culture positives and had pus cell counts (1-5/hpf). However, cases which have more than 5 pus cells/ hpf were all culture positives. This correlated with the study conducted by Subedi $\mathrm{M}$ et al who also showed $81.3 \%$ positive culture isolates in patients who had pus cell count $(1-5 / \mathrm{hpf})$ and $100 \%$ culture positivity in patients with more than 5 pus cells/ hpf. ${ }^{12}$

In the present study, out of the 520 clinically suspected UTI cases, $232(44.61 \%)$ was culture positive. Similar incidence is observed in a study conducted by Subedi $\mathrm{M}$ and Basnyat SR, which showed the percentage of culture positives as $45 \% .{ }^{12}$ Out of the culture positive cases; E.coli was the most common accounting for a total of 144 cases $(80 \%)$. Similarly, Staphylococcous aureus was the most common amongst the Gram positive organisms- 29 cases $(55 \%)$. Nitrofurantoin was found to be the most effective drug against the Gram negative bacteria with a sensitivity of $80 \%$. Ampicillin, Amoxycillin and Cloxacillin were found to be the effective agents against Gram positive bacteria. Subedi M and Basnyat SR in their study found Amikacin as the most effective antibiotic against Gram negative organisms with a sensitivity of $64.4 \%$ followed by ciprofloxacin and Nitrofurantoin. ${ }^{12}$

E.coli is the most common cause of UTI, accounting for $80-90 \%$ of cases. It originates from the fecal flora that colonizes the periurethral area (ascending infection). A study conducted by Santosh JF et al. has also stated that the microorganism frequently isolated associated with first infection of UTI has always been and is coliform bacilli followed by Staphlococcus species. ${ }^{12,15,16}$ Similar type of result was found by Latham et al with $60 \%$ of infection by E.coli, $30 \%$ by Staphylococcus saprophyticus and $10 \%$ by Proteus mirabilis. Similarly, Pandey has shown a reduced incidence of E.coli (35.4\%) followed by Coagulase positive staphylococcus - COPS (22.3\%) and Coagulase negative staphylococcus- CONS (Saprophyticus) $-18.8 \%$. Equal percentage of Enterbacter species and Klebsiella were also isolated, $2.1 \%$ each. He also stated that the microorganism frequently isolated with first infection of UTI has always been coliform bacilli followed by staphylococcus species. ${ }^{17,18}$

Hormonal and mechanical changes increase the risk of urinary stasis and vesico-ureteric reflux. These changes along with an already short urethra and difficulty with hygiene due to distended belly increase the frequency of UTI in pregnant women. ${ }^{9}$

Similarly, the untreated infections are associated with low birth weight, prematurity, premature labour, hypertension, pre-eclampsia and amnionitis. A retrospective population based study by Mazor-Dray et al showed that urinary tract infection during pregnancy is independently associated with intrauterine growth restriction, pre-eclampsia, preterm delivery and even caesarian section. ${ }^{19}$

Similar study conducted by Mohammed V Rabat during a period of 18 months in the military hospital, Morocco had found that out of the 147 patients, 45 had features of UTI. 19 patients had acute pyelonephritis $(28.9 \%)$. Out of the 58 patients with uncontrolled Diabetes Mellitus, 13 had UTI and five had risk of premature labour. ${ }^{20}$

Women with risk factors have the additional risks of a first, recurrent or persistent UTI. These women should undergo more frequent screenings. Risk factors include DM, including gestational diabetes, urologic abnormalities (eg. Neurogenic bladder, duplicated collecting systems), pre-pregnancy and antepartum history of UTI prior to initiation of prenatal care and sickle cell haemoglobinopathies. ${ }^{20}$

\section{CONCLUSIONS}

Screening for bacteriuria is recommended among all pregnant women at the first prenatal visit and in the subsequent trimesters of pregnancy. The most effective method is urinalysis followed by culture. In the presence of risk factors for UTI, it is recommended to do urinalysis every month.

Untreated UTI can lead to complications such as pyelonephritis, low birth weight infants, premature delivery and occasional still birth. Therefore, prompt treatment of symptomatic UTI and asymptomatic bacteriuria is required in pregnant women. 


\section{REFERENCES}

1. Dwyer PL, O'Reilly M. Recurrent urinary tract infection in the female. Curr Opin Obstetric Gynecology 2002; 14(5):537-43. http://dx.doi.org/10.1097/00001703-200210000-00016

2. Le J, Briggs GG, McKeown A, Bustillo G. Urinary tract infections during pregnancy. Ann Pharmacotherapy 2004; 38(10):1692-701. http://dx.doi.org/10.1345/aph.1D630

3. Module design at birth: urinary tract infection in pregnant women. Faculty of Medicine Strasbourg 2005.

4. Lee M, Bozzo P, Einarson A, Koren G. Urinary tract infections in pregnancy. Canadian Fam Physician 2008; 54: 853-4.

5. Gilstrap LC 3rd, Ramin SM. Urinary tract infections during pregnancy. Obstetric Gynecology Clin North Am 2001; 28(3):581-91. http://dx.doi.org/10.1016/S0889$\underline{8545(05) 70219-9}$

6. D'Ercole C, Blanc B. Urinary tract infections during pregnancy: Diagnosis, evolution, prognosis, treatment. Rev Prat 1994; 44(8):1097-103.

7. MacLean $\mathrm{AB}$. Urinary tract infection in pregnancy. Int J Antimicrobial Agents 2001; 17:273-7. http://dx.doi. org/10.1016/S0924-8579(00)00354-X

8. Hill JB, Sheffield JS, McIntire DD, Wendel GD. Acute pyelonephritis in pregnancy. Obstetric Gynecology 2005; 105(1):18-23. http://dx.doi.org/10.1097/01.AOG. $0000149154.96285 . \mathrm{a} 0$

9. Ovalle A, Levancini M. Urinary tract infections in pregnancy. Curr Opin Urol 2001; 11 (1):55-9. http://dx.doi. org/10.1097/00042307-200101000-00008

10. Whalley PJ, Martin FG, Peters PC. Significance of asymptomatic bacteriuria detected during pregnancy. JAMA 1965 Sep 13; 193: 879-81. http://dx.doi.org/10.1001/ jama.1965.03090110017004

11. Kass EH. Maternal urinary tract infection. J New York State Med. 1962; 2882-26.
12. Subedi M, Basnyat SR. Urinary tract infection in Pregnancy and its correlation with Nitrite test, J Nepal Health Res. Council 2009 Oct: 7(15): 80-83

13. Nicolle LE. Asymptomatic bacteriuria: when to screen and when to treat. Infect Dis Clin North Am 2003; 17(2):367-94. http://dx.doi.org/10.1016/S0891-5520(03)00008-4

14. Vazquez JC, Villar J. Treatments for symptomatic urinary tract infections during pregnancy. Cochrane Database System Rev 2003;4: CD002256 http://dx.doi.org/10.1002/14651858. cd002256

15. De Mouy.D, Fabre. R UTI community of women 15 to 65 : sensitivity to antibiotics of E. coli according to history: study AFORCOPI-BIO 2003 Medicine and Infectious Diseases $2007 ; 37: 594-8$.

16. Larabi K, Masmoudi A, Fendri C. Bacteriological and susceptibility study of 1,930 strains isolated from UTIs in a Tunis university hospital. Med Infect Dis 2003;33:348-52

17. Latham RH, Running $\mathrm{K}$, Stamm WE. Urinary tract infection in young adult women caused by Staphylococcus saprophyticus, JAMA. 1983 Dec 9; 250(22):3063-6. http:// dx.doi.org/10.1001/jama.250.22.3063. http://dx.doi. org/10.1001/jama.1983.03340220031028.

18. Pandey RA, Pokharel BM. Hospital based study of Urinary tract infection among women visiting antenatal clinic of TUTH. A dissertation submitted to central department of microbiology, TU, Kathmandu, Nepal 2002.

19. Chung PK, Hall MH. Antenatal prediction of urinary tract infection in pregnancy. Br J Obstetric Gynecology 1982 Jan; 89:8-11. http://dx.doi.org/10.1111/j.1471-0528.1982. $\underline{\text { tb04625.x }}$

20. Bahadur A, El Kalbaj D et al. Urinary tract infection in pregnancy. Saudi J Kidney Dis Transp 2010 Jul 28; 21:342-4. 